\title{
Symmetric hyperbolicity and consistent boundary conditions for second-order Einstein equations
}

\author{
Carsten Gundlach $*$ \\ School of Mathematics, University of Southampton, Southampton SO17 1BJ, UK \\ José M. Martín-Garcídi \\ Instituto de Matemáticas y Física Fundamental, Centro de Física Miguel A. Catalán, \\ C.S.I.C., Serrano 113 bis, 28006 Madrid, Spain
}

(Dated: 3 March 2004)

\begin{abstract}
We present two families of first-order in time and second-order in space formulations of the Einstein equations (variants of the Arnowitt-Deser-Misner formulation) that admit a complete set of characteristic variables and a conserved energy that can be expressed in terms of the characteristic variables. The associated constraint system is also symmetric hyperbolic in this sense, and all characteristic speeds are physical. We propose a family of constraint-preserving boundary conditions that is applicable if the boundary is smooth with tangential shift. We conjecture that the resulting initial-boundary value problem is well-posed.
\end{abstract}

\section{INTRODUCTION}

Current numerical relativity codes designed to simulate the inspiral and merger of a black hole binary are limited by instabilities. These are now believed to be usually instabilities of the continuum equations, rather than of the numerical method. Intuitively one feels that the initial value problem for the Einstein equations is well-posed in a geometrical sense. For instance, in fully harmonic spacetime coordinates the Einstein equations reduce to ten quasilinear wave equations [1]. However, in the usual $3+1$ split one uses only six of the ten Einstein equations for evolution, while the other four must be imposed as constraints on the initial data. An evolution in which the constraints are not obeyed is not a solution of the Einstein equations.

Consider perturbing a solution of the evolution equations around a solution of the full Einstein equations. We call a linear perturbation physical if it obeys the constraints initially and therefore at all times, and if it cannot be removed by a change of coordinates. A perturbation is called pure gauge if it obeys the constraint but can be removed by a coordinate transformation. A perturbation is called unphysical if it violates the constraints. All of these perturbations can be instabilities in the (weak) sense that they grow with respect to the background solution. An example for a physical instability is one that pushes a marginally stable star over the edge of gravitational collapse. An example of a gauge instability is given by the evolution of Minkowski spacetime with constant lapse, with an initial slice that is not quite flat: the time slices become singular in a finite time. Some, perhaps all, gauge instabilities can be avoided by a suitable choice of the lapse and shift adapted to the solution.

*C.Gundlach@maths.soton.ac.uk

†jmm@imaff.cfmac.csic.es
The problem in numerical solutions are unphysical instabilities. These will always be triggered in a numerical simulation by finite differencing or round-off error. Furthermore, the solution space of the evolution equations is infinitely bigger than the solution space of the full Einstein equations, and so the latter are likely to represent an unstable equilibrium. Evolution schemes which replace between one and four of the evolution equations with constraint equations (constrained evolution) do not fundamentally address this problem, as they are still only solving six equations.

In discussing instabilities, it is important to distinguish between evolution systems which are well-posed and evolution systems which are ill-posed. In the former, the growth of any linear perturbation $\delta u(x, t)$ of a background solution $u_{0}(x, t)$ can be bounded as

$$
\|\delta u(\cdot, t)\| \leq f(t)\|\delta u(\cdot, 0)\|,
$$

where $f(t)$ depends on $u_{0}$ but is independent of $\delta u(x, 0)$. This means that the solution $u_{0}+\delta u$ depends continuously on its initial data. By contrast, in an ill-posed system no such bound $f(t)$ exists. Rather, the growth rate increases unboundedly with the highest spatial frequency present in $\delta u(x, 0)$. These are instabilities in a rather stronger sense. They can be gauge, but typically are constraint-violating.

A numerical simulation inherits all the instabilities already present in the continuum. A good numerical scheme does not add any others, but can never fix continuum instabilities. A crucial point is that in a numerical evolution, the highest spatial frequency present is effectively always the grid frequency, because it is generated by finite differencing error even if the initial data are smooth.

As one increases the resolution in numerical solutions of an ill-posed system, constraint violating instabilities start with smaller amplitude (because they are initialized by finite differencing error), but grow more rapidly (because the finer grid can represent a higher spatial frequency). Therefore they don't converge away. At high 
enough resolution and late enough time this will become apparent as a breakdown of convergence for the entire solution, but one can detect their presence before that by looking at a Fourier transform in space [3]. As these non-convergent instabilities are features of the continuum system they cannot be suppressed by any consistent numerical dissipation.

By contrast, the numerical solution of a well-posed scheme will in general still be swamped by constraintviolating instabilities at late time, but now these start with smaller amplitude and grow at the same rate as one increases resolution. They therefore converge away.

In practice, depending on the system, physical solution, runtime and resolution, either convergent or nonconvergent instabilities can be the dominant source of error. In 3-dimensional simulations in particular, the available resolution is quite limited, and the lack of convergence may not become apparent. The ArnowittDeser-Misner (ADM) formulation of the Einstein equations has for many years been the main formulation used in 3-dimensional simulations, even though it is ill-posed (weakly hyperbolic) 2]. More recently a systematic comparison of high-resolution, long-time evolutions for wellposed and ill-posed (weakly hyperbolic) systems [3] has demonstrated that the breakdown of convergence is really inevitable, and has revived interest in well-posed formulations of the Einstein equations.

This interest has focused on first-order reductions of the Einstein equations, because for general first order evolution systems useful criteria for well-posedness are known [4]. A sufficient and necessary criterion for the initial value problem (with no boundaries, or periodic boundaries) to be well-posed is strong hyperbolicity: this roughly means that the system has a complete set of characteristic variables with real speeds. Symmetric hyperbolicity is another criterion which implies strong hyperbolicity and can be used to obtain a well-posed initialboundary value problem: roughly speaking it means that the principal part of the system admits a conserved energy. Therefore a number of strongly or symmetric hyperbolic first-order reductions of the ADM equations have been suggested over the years to assure wellposedness, see for example [5, 6, 7, 8].In a symmetric hyperbolic formulation of the Einstein equations, the constraints can be dealt with consistently in the initialboundary value problem (see [9] for the full, and [10, 11] for the linearised Einstein equations).

However, first order reductions introduce new, auxiliary, variables and constraints, and so further increase the solution space. One would expect this to give rise to additional constraint-violating instabilities of the convergent type, even if well-posedness rules out the non-convergent type. There is some evidence that this is a real problem [12], which may even outweigh the benefits of hyperbolicity. It would therefore seem preferable to find a system that is symmetric hyperbolic while enlarging the solution space as little as possible, and in particular this should be a second-order system.
In a companion paper [13] we have proposed a definition of symmetric hyperbolicity for second-order systems. This can be used to obtain a well-posed initialboundary value problem in the same way as in firstorder systems, but without enlarging the solution space. Here we show symmetric hyperbolicity for two formulations of the Einstein equations that are variants of the Arnowitt-Deser-Misner (ADM) equations, namely the Baumgarte-Shapiro-Shibata-Nakamura (BSSN) system [14, 15], which is already widely used in numerical relativity [16, 17, 18, 19], and a simpler related system suggested by Nagy, Ortiz and Reula (NOR) [2].

We know of two previous partial results concerning the hyperbolicity of a second-order, ADM-like version of the Einstein equations. In 20] it was shown that a first-order reduction of the BSSN system is strongly hyperbolic, and a variant with some superluminal characteristic speeds is symmetric hyperbolic. It was then noted that the auxiliary constraints associated with the introduction of the first-order auxiliary variables form a closed subsystem of the constraint system. This means that if the auxiliary constraints are obeyed initially, and if suitable boundary conditions are imposed, they are obeyed during the evolution, even if the other constraints are not obeyed. In this sense, the introduction of the auxiliary variables has not enlarged the solution system. In [2] it was shown that the NOR system (in second-order form), and its associated constraint system are strongly hyperbolic, by using a pseudo-differential reduction to first order which also does not enlarge the solution space. This method relies in an essential way on Fourier transforms, and cannot be used to deal with the initial-boundary value problem.

Here we go beyond these two papers in showing symmetric hyperbolicity for the second order BSSN and NOR systems and their associated constraint systems, without any reduction to first order. We do this by defining characteristic variables, finding a conserved positive definite covariant energy, and expressing it in terms of the characteristic variables. We have chosen BSSN and NOR because BSSN is popular with numerical relativists today, whereas NOR seems a simpler version of BSSN that shares all its advantages.

The paper is organised as follows. In Sec II we introduce our method and general notation. In Sec. III we prove symmetric hyperbolicity for the BSSN evolution equations and the associated constraint system, and in Sec. IV we do the same for the NOR system. We take a first look at the initial boundary problem for both systems in Sec. $\nabla$ We propose a family of boundary conditions for a smooth boundary where the shift is tangential to the boundary. We show that there are no arbitrarily rapidly growing modes - this amounts to a strong indication of well-posedness but is short of a proof. Sec. VI contains our conclusions. 


\section{METHOD AND NOTATION}

Here we briefly summarise the relevant notation and methods of [13]. We need to split 3-tensors into their longitudinal and transversal parts with respect to a given direction $n_{i}$. Assume a 3 -metric, say $\gamma_{i j}$, which will be used to raise and lower indices, and let $n^{i}$ be a unit vector with respect to this metric. Then

$$
q_{i}{ }^{j} \equiv \delta_{i}{ }^{j}-n_{i} n^{j}
$$

is the projector into the space transversal to $n_{i}$. A tensor index that has been projected will be denoted by the index $A, B, \ldots$ instead of $i, j, \ldots$ An index $n$ denotes a tensor index contracted with $n_{i}$ or $n^{i}$. A self-explanatory example of this notation is

$$
P^{i} \partial_{i} \equiv P_{n} \partial_{n}+P^{A} \partial_{A}
$$

A pair $q q$ of indices indicates a contraction with $q_{i j}$, and for tensors of rank 2 or higher, we use the convention that they are totally tracefree on their projected indices.

Now consider a system of evolution equations that are second order in space and first order in time. Linearise around a solution, and approximate the backgrounddependent coefficients of the linearised system as constant (frozen). Retain only the principal part of the equations. We call the resulting linear system with constant coefficients strongly hyperbolic if for any given direction $n^{i}$ we can find a complete set of characteristic variables $U$ that obey

$$
\partial_{t} U=\lambda \partial_{n} U+\text { transversal derivatives. }
$$

Here $-\lambda$ is the propagation speed in the $n_{i}$ direction. The $U$ are constructed from $\tilde{u} \equiv\left(u, \partial_{i} u\right)$. This definition has two important consequences: transversal derivatives $\partial_{A} u$ are automatically zero speed variables, and arbitrary multiples of transversal derivatives $\partial_{A} u$ can be added to any characteristic variable. Below we shall find the characteristic variables $U$ in two steps: we first find a set of non-zero speed variables $U^{\prime}$ that do not contain any transversal derivatives, then add transversal derivatives to them until we can express the energy and flux in terms of the modified characteristic variables $U$.

We call the system symmetric hyperbolic if it admits an energy

$$
E=\int_{\Omega} \epsilon d V
$$

where $\epsilon$ is covariant, positive definite, and conserved in the sense that

$$
\partial_{t} \epsilon=\partial_{i} F^{i}
$$

for some flux $F^{i}$, and if we can express $\epsilon$ and $F^{n}$ in terms of characteristic variables.

The simplest example is the wave equation in the form

$$
\begin{aligned}
& \partial_{t} \phi=\Pi, \\
& \partial_{t} \Pi=\partial_{i} \partial^{i} \phi,
\end{aligned}
$$

with $u=(\phi, \Pi)$ and $\tilde{u}=\left(\partial_{i} \phi, \Pi\right)$. The characteristic variables in a given direction $n_{i}$ are

$$
\begin{aligned}
U_{ \pm} & \equiv \Pi \pm \partial_{n} \phi, \\
U_{A} & \equiv \partial_{A} \phi,
\end{aligned}
$$

with speeds $\lambda=( \pm 1,0)$. The covariant energy and flux are

$$
\begin{aligned}
\epsilon & =\Pi^{2}+\partial_{i} \phi \partial^{i} \phi, \\
F^{i} & =2 \Pi \partial^{i} \phi .
\end{aligned}
$$

In terms of characteristic variables they are

$$
\begin{aligned}
\epsilon & =\frac{1}{2}\left(U_{+}^{2}+U_{-}^{2}\right)+U_{A} U^{A}, \\
F^{n} & =\frac{1}{2}\left(U_{+}^{2}-U_{-}^{2}\right) .
\end{aligned}
$$

Therefore

$$
\frac{d E}{d t}=\int_{\partial \Omega} \frac{1}{2}\left(U_{+}^{2}-U_{-}^{2}\right) d S
$$

where $U_{ \pm}$are now the characteristic variables normal to the boundary $\partial \Omega$.

The maximally dissipative boundary condition

$$
U_{+}=\kappa U_{-}+f
$$

with $|\kappa| \leq 1$ and $f$ a given function then guarantees that the growth of $E$ is bounded by $f$ and that $E$ does not grow for $f=0$.

To make the energy positive definite in $\phi$ itself rather than just $\partial_{i} \phi$, one can add a term $\alpha^{2} \phi^{2}$ to $\epsilon$, with $\alpha>0$ constant. With a maximally dissipative boundary condition and $f=0, E$ is then bounded by $E(t) \leq e^{\alpha t} E(0)$.

Finally, allow the linearised system to have variable coefficients (from the non-constant background solution) and a non-principal part. For a finite time interval, the energy is then still bounded as $E(t) \leq K e^{\alpha t}$ for some constants $K$ and $\alpha$, and the linearised initial-boundary value problem remains well-posed. Therefore it is sufficient to establish well-posedness to examine the principal part in the frozen coefficient approximation. On an intuitive level, this is so because the purpose of wellposedness is to rule out instabilities of the non-convergent type, which have a growth rate that grows with spatial frequency. Well-posedness of the linearised problem is a necessary condition for well-posedness of the full nonlinear (quasilinear) problem.

\section{THE BSSN FORMULATION OF THE EINSTEIN EQUATIONS}

\section{A. Field equations}

The BSSN formulation of the Einstein equations (without matter) is obtained from the ADM form of the Einstein equations [21],

$$
\partial_{t} \gamma_{i j}=\mathcal{L}_{\beta} \gamma_{i j}-2 \alpha K_{i j},
$$




$$
\begin{aligned}
\partial_{t} K_{i j}= & \mathcal{L}_{\beta} K_{i j}-D_{i} D_{j} \alpha \\
& +\alpha\left(R_{i j}-2 K_{i l} K^{l}{ }_{j}+K K_{i j}\right), \\
H \equiv & R-K_{i j} K^{i j}+K^{2}=0, \\
M_{i} \equiv & D_{j} K^{j}{ }_{i}-D_{i} K=0,
\end{aligned}
$$

by introducing the new variables

$$
\begin{aligned}
\tilde{\gamma}_{i j} & \equiv(\operatorname{det} \gamma)^{-1 / 3} \gamma_{i j} \\
\tilde{\Gamma}^{i} & \equiv \tilde{\gamma}^{i j} \tilde{\gamma}^{k l} \tilde{\gamma}_{j k, l} \\
\phi & \equiv \frac{1}{12} \ln \operatorname{det} \gamma \\
\tilde{A}_{i j} & \equiv(\operatorname{det} \gamma)^{-1 / 3}\left(K_{i j}-\frac{1}{3} \gamma_{i j} K\right)
\end{aligned}
$$

In the remainder of this Section, indices are moved with $\tilde{\gamma}_{i j}$ and its inverse $\tilde{\gamma}^{i j}$. Generalising the BSSN equations, we densitise the lapse $\alpha$ with the determinant of the metric as

$$
\alpha=e^{6 \sigma \phi} Q
$$

where $\sigma$ is a constant and now $Q$, rather than $\alpha$, is a given function of the coordinates. The definition of the $\widetilde{\Gamma}^{i}$ gives rise to the differential constraint

$$
G_{i} \equiv \tilde{\gamma}_{i j} \tilde{\Gamma}^{j}-\tilde{\gamma}^{j k} \tilde{\gamma}_{i j, k}=0
$$

The definition of $\tilde{A}_{i j}$ gives rise to the algebraic constraint

$$
T \equiv \tilde{\gamma}^{i j} \tilde{A}_{i j}=0
$$

and from the definition of $\tilde{\gamma}_{i j}$ we have the algebraic constraint

$$
D \equiv \ln \operatorname{det} \tilde{\gamma}=0
$$

The BSSN equations are first order in time, second order in space, and quasilinear. The principal part of the evolution equations for $\tilde{A}_{i j}, K$ and $\tilde{\Gamma}^{i}$ is given by the highest spatial derivatives, $\left(\partial^{2} \phi, \partial^{2} \tilde{\gamma}, \partial \tilde{A}, \partial K, \partial \tilde{\Gamma}\right)$. The evolution equations for $\tilde{\gamma}$ and $\phi$ do not contain any of these highest derivatives. We define their principal part to be given by the next highest derivatives, that is $(\partial \phi, \partial \tilde{\gamma}, \tilde{A}, K, \tilde{\Gamma})$. Note that with this definition of the principal part the equations are still quasilinear, because the evolution equation for $\gamma_{i j}$ is linear in $K_{i j}$. Note also that $\beta^{k} \partial_{k}$ is part of the principal part of the equations while $\partial \beta$ terms are not. We define the derivative operator

$$
\partial_{0} \equiv \alpha^{-1}\left(\partial_{t}-\beta^{k} \partial_{k}\right)
$$

It is the derivative along the unit vector field normal to the slices of constant time. The principal part of the evolution equations is then

$$
\begin{aligned}
\partial_{0} \phi & \simeq-\frac{1}{6} K \\
\partial_{0} \tilde{\gamma}_{i j} & \simeq-2 \tilde{A}_{i j},
\end{aligned}
$$

$$
\begin{aligned}
\partial_{0} K \simeq & -6 \sigma e^{-4 \phi} \tilde{\gamma}^{i j} \phi_{, i j} \\
\partial_{0} \tilde{A}_{i j} \simeq & e^{-4 \phi}\left[-\frac{1}{2} \tilde{\gamma}^{m n} \tilde{\gamma}_{i j, m n}-2(1+3 \sigma) \phi_{, i j}\right. \\
& \left.+a \tilde{\gamma}_{k(i} \tilde{\Gamma}_{, j)}^{k}+(1-a) \tilde{\gamma}^{k l} \tilde{\gamma}_{k(i, j) l}\right]^{\mathrm{TF}} \\
& -\frac{c}{6} e^{-4 \phi} \tilde{\gamma}_{i j} \tilde{\gamma}^{m n} \tilde{\gamma}^{k l} \tilde{\gamma}_{k l, m n}, \\
\partial_{0} \tilde{\Gamma}^{i} \simeq & 2(b-1) \tilde{\gamma}^{i j} \tilde{\gamma}^{k l} \tilde{A}_{j k, l}-\frac{4}{3} b \tilde{\gamma}^{i j} K_{, j},
\end{aligned}
$$

where $\simeq$ means equal up to non-principal terms, and TF indicates the tracefree part. We have added the constraints $a G_{(i, j)}, 2 b M_{i}$ and $-(c / 6) e^{-4 \phi} \tilde{\gamma}_{i j} \tilde{\gamma}^{k l} D_{, k l}$ to the field equations with free coefficients $a, b$ and $c$. Adding these terms changes the evolution off the constraint surface which can affect the hyperbolicity of the system.

The principal part of the Hamiltonian and momentum constraints is

$$
\begin{aligned}
H & \equiv H_{0}+e^{-4 \phi} \tilde{\gamma}^{i j}\left[a^{\prime} G_{i, j}-\frac{c^{\prime}}{2} D_{, i j}\right], \\
H_{0} & \simeq e^{-4 \phi} \tilde{\gamma}^{i j}\left(\tilde{\gamma}^{k l} \tilde{\gamma}_{k i, j l}-8 \phi_{, i j}\right), \\
M_{i} & \simeq \tilde{A}_{i j, k} \tilde{\gamma}^{j k}-\frac{2}{3} K_{, i} .
\end{aligned}
$$

Here $a^{\prime}$ and $c^{\prime}$ parameterise different ways of writing the Hamiltonian constraint that are found in the literature. We shall work explicitly only with $H_{0}$, and so $a^{\prime}$ and $c^{\prime}$ will not appear below. There are many versions of the BSSN equations which vary in small details in both the principal and non-principal parts. For comparison, the principal part of the version given in 22] is characterised by $\sigma=0$ (the lapse is not densitised) and $a=b=a^{\prime}=$ $c^{\prime}=1, c=0$.

The constraints are compatible with the evolution equations, which means that they form a closed evolution system. It is

$$
\begin{aligned}
\partial_{0} H_{0} \simeq & -2 e^{-4 \phi} \tilde{\gamma}^{i j} M_{i, j}, \\
\partial_{0} M_{i} \simeq & \frac{1}{6} H_{0, i}+e^{-4 \phi}\left(\frac{a}{2} \tilde{\gamma}^{j k} G_{i, j k}\right. \\
& \left.+\frac{a}{6} \tilde{\gamma}^{j k} G_{j, i k}+\frac{1-c}{6} \tilde{\gamma}^{j k} D_{, i j k}\right), \\
\partial_{0} G_{i}= & 2 b M_{i}, \\
\partial_{0} T \simeq & -\frac{c}{2} e^{-4 \phi} \tilde{\gamma}^{i j} D_{, i j}, \\
\partial_{0} D= & -2 T .
\end{aligned}
$$

\section{B. Strong hyperbolicity of the main system}

For the purpose of decomposing 3-tensors and tensor equations, we define $n_{i}, n^{i}$ and $q_{i j}$ with respect to the conformal metric $\tilde{\gamma}_{i j}$. In the frozen coefficients approximation, the undifferentiated 3-metric $\left(\tilde{\gamma}_{i j}, \tilde{\gamma}^{i j}\right.$ and $\left.\phi\right)$ is to be treated as a background quantity, while $\tilde{A}_{i j}, \tilde{\Gamma}^{i}$, $K, \tilde{\gamma}_{i j, k}, \phi_{, i}$ and their derivatives are to be treated as dynamical variables, and decomposed with respect to $n_{i}$. 
We now prove strong hyperbolicity of the main and constraint systems by constructing a complete set of characteristic variables $U$. The quantities

$$
\tilde{u} \equiv\left(\partial_{i} \phi, \partial_{i} \tilde{\gamma}_{j k}, \tilde{A}_{i j}, \tilde{\Gamma}^{i}, K\right) .
$$

obey the pseudo-first order system

$$
\partial_{t} \tilde{u} \simeq\left(\alpha P^{i}+\beta^{i}\right) \partial_{i} \tilde{u}
$$

or equivalently

$$
\partial_{0} \tilde{u} \simeq P^{i} \partial_{i} \tilde{u} .
$$

(This is not a genuine first-order system because in expressions like $\partial_{i}\left(\partial_{j} \phi\right)$ that appear on its right-hand side we allow ourselves to commute partial derivatives, rather than treating $\partial_{j} \phi$ as a 1 -form variable $d_{j}$, as we would in a genuine reduction to first order.) As discussed in [13], transversal derivatives are automatically zero speed characteristic variables. Here we have $P_{n} \partial_{A} \phi=P_{n} \partial_{A} \tilde{\gamma}_{i j}=0$. We shall write the eigenvalues of $P_{n}$ as $\lambda e^{-2 \phi}$. With this definition $\lambda$ measures the propagation speed in units of the speed of light, measured with respect to the $\partial_{0}$ observers. In particular $\lambda= \pm 1$ variables propagate along the light cone.

In tensor components with respect to $n_{i}$, the scalar block of $P_{n}$ is given by

$$
\begin{aligned}
P_{n} \partial_{n} \phi= & -\frac{1}{6} K \\
P_{n} \partial_{n} \tilde{\gamma}_{n n}= & -2 \tilde{A}_{n n}, \\
P_{n} \partial_{n} \tilde{\gamma}_{q q}= & -2 \tilde{A}_{q q}, \\
P_{n} K= & -6 \sigma e^{-4 \phi} \partial_{n} \phi, \\
P_{n} \tilde{A}_{n n}= & e^{-4 \phi}\left[-\left(\frac{4}{3}+4 \sigma\right) \partial_{n} \phi+\frac{1}{6} \partial_{n} \tilde{\gamma}_{n n}\right. \\
& +\frac{2 a}{3}\left(\tilde{\Gamma}_{n}-\partial_{n} \tilde{\gamma}_{n n}\right) \\
& \left.+\frac{1-c}{6}\left(\partial_{n} \tilde{\gamma}_{n n}+\partial_{n} \tilde{\gamma}_{q q}\right)\right], \\
P_{n} \tilde{A}_{q q}= & e^{-4 \phi}\left[\left(\frac{4}{3}+4 \sigma\right) \partial_{n} \phi-\frac{1}{6} \partial_{n} \tilde{\gamma}_{n n}\right. \\
& -\frac{2 a}{3}\left(\tilde{\Gamma}_{n}-\partial_{n} \tilde{\gamma}_{n n}\right) \\
& \left.-\frac{1+2 c}{6}\left(\partial_{n} \tilde{\gamma}_{n n}+\partial_{n} \tilde{\gamma}_{q q}\right)\right], \\
& -\frac{4 b}{3} K+2(b-1) \tilde{A}_{n n} .
\end{aligned}
$$

The two algebraic constraints $T=0$ and $D=0$ are often enforced in numerical simulations after each time step. We can mimic enforcing $T=0$ in our analysis by setting $\tilde{A}_{n n}+\tilde{A}_{q q}=0$ and dropping one of the two variables from the system. Similarly, we can mimic enforcing $D=0$ by setting $\partial_{n} \tilde{\gamma}_{n n}+\partial_{n} \tilde{\gamma}_{q q}=0$ and dropping one of the two variables from the system. Both reductions affect the hyperbolicity of the system. a. Algebraic constraints not enforced The scalar block is diagonalisable for

$$
c>0, \quad \eta>0, \quad \sigma>0, \quad c \neq \eta,
$$

with eigenvalues

$$
\lambda=\{0, \pm \sqrt{c}, \pm \sqrt{\eta}, \pm \sqrt{\sigma}\},
$$

where we have defined the shorthand

$$
\eta \equiv \frac{4 a b-1}{3} \text {. }
$$

(Allowing the system to be strongly hyperbolic without enforcing the algebraic constraints is the reason why we have introduced the $c$ term, which potentially gives $\partial_{0} \tilde{A}_{i j}$ a non-zero trace.)

b. Trace constraint enforced If we enforce $T=0$ but not $D=0$ this is consistent with the evolution equations only for $c=0$. The scalar block is diagonalisable for

$$
c=0, \quad \eta>0, \quad \sigma>0,
$$

with

$$
\lambda=\{0,0, \pm \sqrt{\eta}, \pm \sqrt{\sigma}\} .
$$

It would be inconsistent to enforce $D=0$ but not $T=0$.

c. Both algebraic constraints enforced If we enforce both algebraic constraints $D=0$ and $T=0$ the scalar block is diagonalisable for

$$
\eta>0, \quad \sigma>0 .
$$

(Note that $c$ becomes irrelevant in this case.) The characteristic variables that do not contain any transversal derivatives are:

$$
\begin{aligned}
U_{0}^{\prime} \equiv & (b-1) \partial_{n} \tilde{\gamma}_{n n}+\tilde{\Gamma}_{n}-8 b \partial_{n} \phi \\
U_{ \pm}^{\prime} \equiv & (1-4 a) \partial_{n} \tilde{\gamma}_{n n}+4 a \tilde{\Gamma}_{n}-8 \partial_{n} \phi \\
& \pm e^{2 \phi} \sqrt{\eta}\left(6 \tilde{A}_{n n}-4 K\right) \\
V_{ \pm}^{\prime} \equiv & 6 \sqrt{\sigma} \partial_{n} \phi \mp e^{2 \phi} K
\end{aligned}
$$

with speeds

$$
\lambda=(0, \pm \sqrt{\eta}, \pm \sqrt{\sigma}) .
$$

The vector block of $P_{n}$ is

$$
\begin{aligned}
P_{n} \partial_{n} \tilde{\gamma}_{A n} & =-2 \tilde{A}_{A n}, \\
P_{n} \tilde{A}_{A n} & =e^{-4 \phi} \frac{a}{2}\left(\tilde{\Gamma}_{A}-\partial_{n} \tilde{\gamma}_{A n}\right), \\
P_{n} \tilde{\Gamma}_{A} & =2(b-1) \tilde{A}_{A n},
\end{aligned}
$$

as well as the trivial $P_{n} \partial_{A} \phi=P_{n} \partial_{A} \tilde{\gamma}_{n n}=P_{n} \partial_{A} \tilde{\gamma}_{q q}=0$. It is diagonalisable for $a b>0$, with nontrivial characteristic variables

$$
\begin{aligned}
U_{A}^{\prime} & \equiv(b-1) \partial_{n} \tilde{\gamma}_{n A}+\tilde{\Gamma}_{A}, \\
U_{ \pm A}^{\prime} & \equiv-a \partial_{n} \tilde{\gamma}_{A n}+a \tilde{\Gamma}_{A} \pm 2 \sqrt{a b} e^{2 \phi} \tilde{A}_{A n},
\end{aligned}
$$


with speeds $\lambda=(0, \pm \sqrt{a b})$. The tensor block is

$$
\begin{aligned}
P_{n} \partial_{n} \tilde{\gamma}_{A B} & =-2 \tilde{A}_{A B}, \\
P_{n} \tilde{A}_{A B} & =-e^{-4 \phi} \frac{1}{2} \partial_{n} \tilde{\gamma}_{A B},
\end{aligned}
$$

as well as the trivial $P_{n} \partial_{A} \tilde{\gamma}_{B n}=0$. It is always diagonalisable, with nontrivial characteristic variables

$$
U_{ \pm A B}^{\prime} \equiv \frac{1}{2} \partial_{n} \tilde{\gamma}_{A B} \mp e^{2 \phi} \tilde{A}_{A B},
$$

with speeds $\lambda= \pm 1$. We see that the vector and tensor sectors do not add any new conditions for strong hyperbolicity.

In summary, we have shown that the BSSN system with both algebraic constraints enforced continuously is strongly hyperbolic if $\sigma>0$ and $\eta>0$. If neither algebraic constraint is enforced, $c>0$ and $c \neq \eta$ are also required. On the other hand, if only the trace constraint is enforced, $c=0$ is required. (If both constraints are enforced, $c$ is irrelevant and can be set to zero.) The characteristic spectrum of the complete system is

$$
\lambda=\{0, \pm \sqrt{\sigma}, \pm \sqrt{\eta}, \pm \sqrt{a b}, \pm 1\} .
$$

\section{Strong hyperbolicity of the constraint system}

We construct characteristic variables for the constraint system from the set

$$
\tilde{u}_{c} \equiv\left(H_{0}, M_{i}, \partial_{i} G_{j}, \partial_{i} T, \partial_{i} \partial_{j} D\right) .
$$

The scalar sector of the constraint propagation is

$$
\begin{aligned}
P_{n} H_{0} & =-2 e^{-4 \phi} M_{n} \\
P_{n} M_{n} & =\frac{1}{6} H_{0}+e^{-4 \phi}\left(\frac{2 a}{3} \partial_{n} G_{n}+\frac{1-c}{6} \partial_{n}^{2} D\right) \\
P_{n} \partial_{n} G_{n} & =2 b M_{n} \\
P_{n} \partial_{n} T & =-\frac{c}{2} e^{-4 \phi} \partial_{n}^{2} D \\
P_{n} \partial_{n}^{2} D & =-2 \partial_{n} T
\end{aligned}
$$

If we define

$$
C_{ \pm}^{\prime} \equiv 4 a \partial_{n} G_{n} \pm 6 \sqrt{\eta} e^{2 \phi} M_{n}+e^{4 \phi} H_{0}
$$

the characteristic variables are

$$
\begin{array}{r}
C_{0}^{\prime} \equiv e^{4 \phi} b H_{0}+\partial_{n} G_{n}, \\
\sqrt{c} \partial_{n}^{2} D \mp 2 e^{2 \phi} \partial_{n} T, \\
(1-c)\left(\partial_{n}^{2} D \mp \frac{2}{\sqrt{\eta}} e^{2 \phi} \partial_{n} T\right)+\left(1-\frac{c}{\eta}\right) C_{ \pm}^{\prime},
\end{array}
$$

with speeds $\lambda=(0, \pm \sqrt{c}, \pm \sqrt{\eta})$. If we optionally impose the trace constraint, the characteristic variables are $C_{0}^{\prime}$ and $C_{ \pm}^{\prime}+\partial_{n}^{2} D$ with speeds $\lambda=(0, \pm \sqrt{\eta})$. If we impose both the trace and determinant constraints, we are left with $C_{0}^{\prime}$ and $C_{ \pm}^{\prime}$ with speeds $\lambda=(0, \pm \sqrt{\eta})$.
The vector sector of the constraint propagation is

$$
\begin{aligned}
P_{n} M_{A} & =e^{-4 \phi} \frac{a}{2} \partial_{n} G_{A}, \\
P_{n} \partial_{n} G_{A} & =2 b M_{A},
\end{aligned}
$$

as well as $P_{n} \partial_{A} G_{n}=P_{n} \partial_{A} T=P_{n} \partial_{A} \partial_{n} D=0$. The nontrivial characteristic variables are

$$
C_{ \pm A}^{\prime} \equiv a \partial_{n} G_{A} \pm 2 \sqrt{a b} e^{2 \phi} M_{A} .
$$

with speeds $\lambda= \pm \sqrt{a b}$. The tensor sector is completely trivial, with $P_{n} \partial_{A} G_{B}=P_{n} \partial_{A} \partial_{B} T=0$ (including the traces). The constraint system, in all three cases, is strongly hyperbolic as long as the main system is strongly hyperbolic.

\section{Main system energy}

We look for an energy density $\epsilon$ of the second-order system that is positive definite in $\tilde{A}_{i j}, K, \tilde{\Gamma}_{i}, \tilde{\gamma}_{i j, k}$ and $\phi_{, i}$ and obeys a conservation law. With the shorthands $t_{i} \equiv \tilde{\gamma}^{j k} \tilde{\gamma}_{j k, i}=D_{, i}$ and $d_{i} \equiv \tilde{\gamma}^{j k} \tilde{\gamma}_{i j, k}$, the most general quadratic form in these variables is

$$
\begin{aligned}
\epsilon= & c_{0} e^{4 \phi} \tilde{A}_{i j}{ }^{2}+c_{1} \tilde{\Gamma}_{i}{ }^{2}+c_{2} t_{i}{ }^{2}+c_{3} d_{i}{ }^{2} \\
& +c_{4} d_{i} \tilde{\Gamma}^{i}+c_{5} t_{i} \tilde{\Gamma}^{i}+c_{6} d_{i} t^{i}+c_{7} e^{4 \phi} K^{2} \\
& +c_{8} \tilde{\gamma}_{i j, k}{ }^{2}+c_{9} \tilde{\gamma}_{i j, k} \tilde{\gamma}^{i k, j}+c_{10} \tilde{\Gamma}_{i} \phi^{, i}+c_{11} e^{4 \phi} T K \\
& +c_{12} \phi_{, i}{ }^{2}+c_{13} d_{i} \phi^{, i}+c_{14} t_{i} \phi^{, i}+c_{15} e^{4 \phi} T^{2} .
\end{aligned}
$$

In the cross term $\tilde{\gamma}_{i j, k} \tilde{\gamma}^{i k, j}$ and similar terms in the remainder of the paper indices are raised only after differentiation. The general ansatz for the flux $F^{i}$ contains 14 free coefficients. After some linear algebra it is possible to see that this $\epsilon$ and $F^{i}$ together obey a conservation law only if

$$
4 a b=1+3 \sigma
$$

or equivalently $\eta=\sigma$.

There are additional restrictions depending on whether or not we enforce the algebraic constraints during evolution. If we work with non-vanishing $D$ and $T$ then we need $a b=\sigma=1$ and $c_{11}(c-1)=0$. On the other hand, if we enforce both algebraic constraints the parameter $c$ and several of the coefficients $c_{k}$ become irrelevant, with no additional constraints on the parameters.

From now on, in order to simplify the equations, we enforce both the $T=0$ and $D=0$ constraints in the remainder of this Section.

The generic conserved energy depends on four free coefficients, one of which is an overall factor. With the shorthands

$$
\begin{aligned}
\epsilon_{0}= & e^{4 \phi} \tilde{A}_{i j}^{2}+\frac{1}{4} \tilde{\gamma}_{i j, k}{ }^{2}+\frac{2 a-a b-1}{2} \tilde{\gamma}_{i j, k} \tilde{\gamma}^{i k, j} \\
& -a d_{i}\left(\tilde{\Gamma}^{i}-8 b \phi^{, i}\right),
\end{aligned}
$$




$$
\begin{aligned}
\epsilon_{1} & =\left[\tilde{\Gamma}_{i}-8 b \phi_{, i}+(b-1) d_{i}\right]^{2}, \\
\epsilon_{2} & =d_{i}{ }^{2}-\tilde{\gamma}_{i j, k} \tilde{\gamma}^{k i, j}, \\
\epsilon_{3} & =e^{4 \phi} K^{2}+36 \sigma \phi^{, i} \phi_{, i}
\end{aligned}
$$

the energy density is

$$
\epsilon=c_{0} \epsilon_{0}+c_{1} \epsilon_{1}+\left[c_{3}-(b-1)^{2} c_{1}\right] \epsilon_{2}+c_{7} \epsilon_{3} .
$$

The free parameters $c_{0}, c_{1}, c_{3}$ and $c_{7}$ will be restricted below by inequalities derived from the requirement that $\epsilon$ be positive definite.

This energy reduces to that given in [20] for a firstorder reduction of BSSN with the change of notation $\sigma \rightarrow 2 \sigma, b \rightarrow m, \tilde{\gamma}_{i j, k} \rightarrow d_{k i j}$ and $\phi_{, i} \rightarrow d_{i} / 12$, the restriction $a=1$ to the parameters of the evolution equations, fixing the overall scale as $c_{0}=1$, and the further restrictions $c_{1}=1 /(2 b-2), c_{3}=0$ and $c_{7}=1 / \sigma=3 /(4 b-1)$ on the coefficients of the energy. The condition (86) on $\sigma$ reduces to a similar condition in 20]. The choice of Sarbach et al. can be interpreted as follows: $c_{3}=0$ and $c_{1}=a /(2 b-2)$ eliminate $d_{i}$ from the energy $\left(c_{3}=c_{4}=c_{13}=0\right)$; assuming those conditions, $a=1$ is the only choice that eliminates the cross term $\tilde{\gamma}_{i j, k} \tilde{\gamma}^{k i, j}$. Their choice for $c_{7}$ has no relevant effect. Note that the positivity condition on their choice of $c_{1}$ forces $b>1$, and together with $a=1$ this gives rise to a superluminal speed. By contrast, by retaining the contractions $t_{i}$ and $d_{i}$ in the ansatz for the energy we shall be able to make all speeds physical.

The four terms in the energy are conserved separately:

$$
\begin{aligned}
& \partial_{0} \epsilon_{0}=\partial_{i} F_{0}^{i}, \\
& \partial_{0} \epsilon_{1}=0, \\
& \partial_{0} \epsilon_{2}=\partial_{i} F_{2}^{i}, \\
& \partial_{0} \epsilon_{3}=\partial_{i} F_{3}^{i} .
\end{aligned}
$$

(Condition (86) is required only for the conservation of $\epsilon_{0}$.) The fluxes are given by

$$
\begin{aligned}
F_{0}^{i}= & 2 \tilde{A}^{i j}\left[a \tilde{\Gamma}_{j}-a(b-1) d_{j}-2(1+3 \sigma) \phi_{, j}\right] \\
& +\tilde{\gamma}^{i l} \tilde{A}^{j k}\left[2(1+a b-2 a) \tilde{\gamma}_{j l, k}-\tilde{\gamma}_{j k, l}\right], \\
F_{2}^{i}= & 4 \tilde{A}^{j k}\left(\tilde{\gamma}^{i m} \tilde{\gamma}_{m j, k}-\tilde{\gamma}^{i}{ }_{j} \tilde{\gamma}_{k m}{ }^{, m}\right), \\
F_{3}^{i}= & -12 \sigma K \phi^{, i} .
\end{aligned}
$$

The energy density in terms of characteristic variables is

$$
\begin{aligned}
\epsilon= & \frac{c_{0}}{2}\left(U_{+A B}^{2}+U_{-A B}^{2}\right)+\frac{c_{0}}{4 a b}\left(U_{+A}^{2}+U_{-A}^{2}\right) \\
& +\frac{c_{0} c_{7}}{16 \sigma\left(2 c_{0}+3 c_{7}\right)}\left(U_{+}^{2}+U_{-}^{2}\right)+\frac{2 c_{0}+3 c_{7}}{6}\left(V_{+}^{2}+V_{-}^{2}\right) \\
& + \text { quadratic in zero speed variables, }
\end{aligned}
$$

and the flux is

$$
\begin{aligned}
e^{2 \phi} F^{n}= & \frac{c_{0}}{2}\left(U_{+A B}^{2}-U_{-A B}^{2}\right)+\frac{c_{0}}{4 \sqrt{a b}}\left(U_{+A}^{2}-U_{-A}^{2}\right) \\
& +\frac{c_{0} c_{7}}{16 \sqrt{\sigma}\left(2 c_{0}+3 c_{7}\right)}\left(U_{+}^{2}-U_{-}^{2}\right) \\
& +\frac{2 c_{0}+3 c_{7}}{6} \sqrt{\sigma}\left(V_{+}^{2}-V_{-}^{2}\right)
\end{aligned}
$$

We have modified the characteristic variables by adding terms in $\partial_{A} \tilde{\gamma}_{i j}$ in order to write $\epsilon$ in terms of characteristic variables. With the shorthands

$$
\begin{aligned}
& z_{1}=\frac{c_{3}-(b-1)^{2} c_{1}}{c_{0}}, \\
& z_{2}=1+3 a b-4 a+6 z_{1}, \\
& z_{3}=a-a b-2 z_{1}, \\
& z_{4}=2+3 a b-5 a+6 z_{1}, \\
& z_{5}=1+a b-2 a+2 z_{1}, \\
& z_{6}=\frac{c_{0}}{2 \sqrt{\sigma}\left(2 c_{0}+3 c_{7}\right)},
\end{aligned}
$$

the modified characteristic variables are

$$
\begin{aligned}
U_{ \pm}= & (1-4 a) \partial_{n} g_{n n}+4 a \tilde{\Gamma}_{n}-8 \partial_{n} \phi \\
& \pm \sqrt{\sigma} e^{2 \phi}\left(6 \tilde{A}_{n n}-4 K\right)-2 z_{2} \partial^{A} g_{A n}, \\
V_{ \pm}= & 6 \sqrt{\sigma} \partial_{n} \phi \mp e^{2 \phi} K-z_{6} U_{ \pm} \\
U_{ \pm A}= & \pm 2 \sqrt{a b} e^{2 \phi} \tilde{A}_{A n}+a \tilde{\Gamma}_{A}-a \partial_{n} g_{A n} \\
& +z_{3} \partial^{B} g_{A B}+\frac{z_{4}}{2} \partial_{A} g_{n n}-8 a b \partial_{A} \phi, \\
U_{ \pm A B}= & \mp e^{2 \phi} \tilde{A}_{A B}+\frac{1}{2} \partial_{n} g_{A B} \\
& -\frac{z_{5}}{2}\left(\partial_{A} g_{B n}+\partial_{B} g_{A n}-q_{A B} \partial^{C} g_{C n}\right)
\end{aligned}
$$

The expressions for $U_{0}$ and $U_{A}$ are not given, but they also include transversal derivatives. Note that because $\eta=\sigma, U_{ \pm}^{\prime}$ and $V_{ \pm}^{\prime}$ are eigenvectors for the same eigenvalues, and we have used this to make the energy diagonal in $U_{ \pm}$and $V_{ \pm}$.

\section{E. Constraint system energy}

The constraint energy is quadratic in $H_{0}, M_{i}$ and $G_{i, j}$. (We must use $G_{i, j}$ rather than $G_{i}$ so that all terms are of the same order in derivatives). The most general conserved expression is

$$
\begin{aligned}
\epsilon_{c}= & w_{0} e^{8 \phi} H_{0}^{2}+12 w_{1} e^{4 \phi} M_{i}^{2}+\frac{3 a w_{1}}{b}\left(G_{i, j}\right)^{2} \\
& +w_{2} G_{i, j} G^{j, i}+2 \frac{w_{0}+w_{1}}{b} e^{4 \phi} G_{i}{ }^{i} H_{0} \\
& +\frac{w_{0}+(1+a b) w_{1}-b^{2} w_{2}}{b^{2}}\left(G_{i}{ }^{i}\right)^{2},
\end{aligned}
$$

with arbitrary coefficients $w_{0}, w_{1}$ and $w_{2}$. The corresponding flux is

$$
\begin{aligned}
F_{c}^{i}= & 4 w_{1}\left(e^{4 \phi} H_{0} M^{i}+3 a G^{j, i} M_{j}+a M^{i} G^{j}{ }_{, j}\right) \\
& +4 b w_{2}\left(G^{i, j} M_{j}-M^{i} G^{j}{ }_{, j}\right) .
\end{aligned}
$$

In terms of characteristic variables, the constraint energy and flux are

$$
\epsilon_{c}=\frac{3 w_{1}}{2 a b}\left(C_{-A} C_{-}^{A}+C_{+A} C_{+}^{A}\right)+\frac{w_{1}}{6 \sigma}\left(C_{-}^{2}+C_{+}^{2}\right)
$$


+ quadratic in zero speed variables,

$$
\begin{array}{r}
e^{2 \phi} F_{c}^{n}=\frac{3 w_{1}}{2 \sqrt{a b}}\left(C_{+A} C_{+}^{A}-C_{-A} C_{-}^{A}\right) \\
+\frac{w_{1}}{6 \sqrt{\sigma}}\left(C_{+}^{2}-C_{-}^{2}\right),
\end{array}
$$

where the modified non-zero speed characteristic variables are

$$
\begin{aligned}
C_{ \pm}= & e^{4 \phi} H_{0} \pm 6 e^{2 \phi} \sqrt{\sigma} M_{n}+4 a \partial_{n} G_{n} \\
& +\frac{a w_{1}-b w_{2}}{w_{1}} \partial^{A} G_{A} \\
C_{ \pm A}= & \pm 2 \sqrt{a b} e^{2 \phi} M_{A}+a \partial_{n} G_{A}+\frac{w_{2} b}{3 w_{1}} \partial_{A} G_{n}
\end{aligned}
$$

Note that $w_{2}$ does not appear explicitly in (113) or (114), but it does appear in the definition of the characteristic variables.

\section{F. Symmetric hyperbolicity and causal speeds}

With the condition $\eta=\sigma$ that is required for conservation of the main energy, the complete spectrum can be written entirely in terms of $\sigma$ :

$$
\lambda=\left(0, \pm \sqrt{\sigma}, \pm \frac{\sqrt{1+3 \sigma}}{2}, \pm 1\right) .
$$

Strong hyperbolicity requires $\sigma>0$ and the absence of superluminal speeds requires $\sigma \leq 1$. Note that with $\sigma=$ $a b=1$ all speeds are either 0 or \pm 1 .

Now we impose positive definiteness of the energies, that is, all eigenvalues of their respective matrices are strictly positive. This means that the main energy vanishes if and only if $\tilde{\gamma}_{i j}$ and $\phi$ are constant and all other variables vanish, and that the constraint energy vanishes if and only if $G_{i}$ is constant and the Hamiltonian and momentum constraints vanish. (We are assuming that the two algebraic constraints vanish because they are being continuously re-imposed.)

To see when the constraint energy is positive definite, we need to decompose $G_{i, j}$ as

$$
G_{i, j}=S_{i j}+A_{i j}+\frac{1}{3} \gamma_{i j} G_{, k}^{k}
$$

where $S_{i j}$ is symmetric and tracefree and $A_{i j}$ is antisymmetric. We obtain a sum of simple squares and a quadratic form in the two variables $H_{0}$ and $G^{i}{ }_{i}$.

The constraint energy is positive definite if and only if

$$
\begin{aligned}
& w_{0}>0, \quad 0<w_{1}<3 w_{0}, \quad \frac{w_{0}+w_{1}}{4 w_{0}}<a b \leq 1 \\
& -a b<\frac{w_{2} b^{2}}{3 w_{1}}<a b-\frac{w_{0}+w_{1}}{2 w_{0}} .
\end{aligned}
$$

It is interesting to see how $w_{1}$ affects the possible range for $a b$.
The positivity of the main energy is more complicated. We must take into account that the partial traces $t_{i}$ and $d_{i}$ of the three-index object $\tilde{\gamma}_{i j, k}$ appear both explicitly and explicitly in the energy. We therefore decompose it as

$$
\tilde{\gamma}_{i j, k}=\frac{1}{5}\left[\left(3 d_{(i}-t_{(i}\right) \tilde{\gamma}_{j) k}+\left(2 t_{k}-d_{k}\right) \tilde{\gamma}_{i j}\right]+f_{i j k}
$$

where $f_{i j k}$ is completely tracefree. We then obtain a quadratic form in $t_{i}$ and $d_{i}$, plus $c_{8} f_{i j k}^{2}+c_{9} f_{i j k} f^{i k j}$. To analyse the positivity of the latter we decompose $f_{i j k}$ in its 12 independent frame components and analyse the corresponding quadratic form by brute force. It turns out to be positive if and only if $c_{8}>0$ and $-c_{8}<c_{9}<2 c_{8}$. Finally, assuming causal speeds, we obtain the positivity conditions

$$
\begin{aligned}
& c_{0}>0, \quad c_{7}>0, \quad 2 a^{2} c_{0}<(4 a b-1) c_{1}, \\
& 5 c_{0} \frac{2 a^{2} c_{0}-(4 a b-1) c_{1}}{36 c_{1}} \\
& <c_{3}-(b-1)^{2} c_{1}+\frac{1+2 a b-4 a}{4} c_{0}<0 .
\end{aligned}
$$

The three sets of inequalities are compatible, and can be solved sequentially, in this order: first choose $c_{0}, c_{7}$, $w_{0}$ and $w_{1}$ independently, then $a$ and $b$, followed by $c_{1}$. Finally choose $c_{3}$ and $w_{2}$. It is clear from this construction that the solutions form a single connected set. For example, a possible solution with $\lambda=(0, \pm 1)$ is

$$
\begin{aligned}
& a=b=\sigma=1, \\
& c_{0}=c_{7}=1, \quad c_{1}=2, \quad c_{3}=0 \\
& w_{0}=1, \quad w_{1}=\frac{1}{2}, \quad w_{2}=0 .
\end{aligned}
$$

\section{THE NOR FORMULATION OF THE EINSTEIN EQUATIONS}

\section{A. Field equations}

In this Section, all indices are moved with $\gamma_{i j}$ and its inverse $\gamma^{i j}$. The NOR system is obtained from the ADM system with densitised lapse by introducing the variables

$$
f_{i} \equiv \gamma_{i j}{ }^{j}-\frac{\rho}{2} \gamma^{j k} \gamma_{j k, i}
$$

where $\rho$ is a constant parameter. In [2] the choice $\rho=1$ is made. $\rho=2 / 3$ makes $f_{i}=\tilde{\gamma}_{i j} \tilde{\Gamma}^{j}$. The definition of $f_{i}$ gives rise to the constraint

$$
G_{i} \equiv f_{i}-\gamma_{i j}{ }^{j}+\frac{\rho}{2} \gamma^{j k} \gamma_{j k, i}=0
$$

As in the BSSN system, we parameterise the use of this constraint with $a$ and the use of the momentum constraint with $b$ by adding $a G_{(i, j)}$ and $2 b M_{i}$ to the evolution equations for $K_{i j}$ and $f_{i}$. Following [2], we also add 
$c \gamma_{i j} H$ to the evolution equation for $K_{i j}$ with free parameter $c$. The principal part of the evolution equations is

$$
\begin{aligned}
\partial_{0} \gamma_{i j} \simeq & -2 K_{i j}, \\
\partial_{0} K_{i j} \simeq & \frac{1}{2}\left[a\left(f_{i, j}+f_{j, i}\right)-\gamma_{i j, k}{ }^{,}\right. \\
& +(1-a)\left(\gamma_{k i, j}{ }^{,}{ }^{k}+\gamma_{k j, i}{ }^{k}\right) \\
& \left.+(a \rho-1-\sigma) \gamma^{k l} \gamma_{k l, i j}\right]+c \gamma_{i j} H, \\
\partial_{0} f_{i} \simeq & 2(b-1) K_{i j}{ }^{j}+(\rho-2 b) K_{, i},
\end{aligned}
$$

where

$$
\begin{aligned}
H & \equiv H_{0}+a^{\prime} G_{i}{ }^{i}, \\
H_{0} & \simeq \gamma_{i j}{ }^{i j}-\gamma^{i j} \gamma_{i j, k}{ }^{, k} \\
M_{i} & \simeq K_{i j}{ }^{j}-K_{, i} .
\end{aligned}
$$

In [2], $\rho=a=a^{\prime}=1$.

The constraints system is

$$
\begin{aligned}
\partial_{0} H_{0} \simeq & -2 M_{i}{ }^{i}, \\
\partial_{0} M_{i} \simeq & -\left(\frac{1}{2}+2 c\right) H_{0, i} \\
& +\frac{a}{2} G_{i, j}{ }^{j}-\left(\frac{a}{2}+2 c a^{\prime}\right) G_{j, i}{ }^{j}, \\
\partial_{0} G_{i}= & 2 b M_{i} .
\end{aligned}
$$

\section{B. Strong hyperbolicity}

This proceeds exactly as in the BSSN system, with pseudo-first order variables

$$
\tilde{u} \equiv\left(\partial_{i} \gamma_{j k}, K_{i j}, f_{i}\right)
$$

and

$$
\tilde{u}_{c}=\left\{H_{0}, M_{i}, \partial_{i} G_{j}\right\}
$$

for the constraint system. We define $n_{i}, n^{i}$ and $q_{i j}$ with respect to $\gamma_{i j}$. (In [2], a flat auxiliary metric is introduced instead.)

The scalar sector of the main system is diagonalisable for $\chi>0, \sigma>0$ and $\chi \neq \sigma$, with

$$
\lambda=\{0, \pm \sqrt{\chi}, \pm \sqrt{\sigma}\},
$$

with the shorthand

$$
\chi \equiv 1+4 c\left(1-a^{\prime} b\right) .
$$

If $\eta=\chi$ we can diagonalise the scalar sector also for $\chi=\sigma$. (Note that while we use the same shorthand $\eta$ defined by (55) as in the BSSN system, $\sqrt{\eta}$ is not a speed in the NOR system.)

The vector sector is diagonalisable for $a b>0$ with

$$
\lambda=\{0, \pm \sqrt{a b}\},
$$

and the tensor sector is always diagonalisable, with $\lambda=$ \pm 1 . For general values of $\left(\rho, \sigma, a, b, c, a^{\prime}\right)$ the characteristic variables are too long to give here.

The scalar sector of the constraint system is diagonalisable for $\chi>0$ with characteristic variables

$$
\begin{array}{r}
C_{0} \equiv \partial_{n} G_{n}+b H_{0}, \\
C_{ \pm} \equiv(1+4 c) H_{0}+4 c a^{\prime} \partial_{n} G_{n} \mp 2 \sqrt{\chi} M_{n},
\end{array}
$$

with speeds $\lambda=\{0, \pm \sqrt{\chi}\}$, and the vector sector is diagonalisable for $a b>0$ with

$$
C_{ \pm A} \equiv a \partial_{n} G_{A} \pm 2 \sqrt{a b} M_{A},
$$

with speeds $\lambda=\{ \pm \sqrt{a b}\}$.

The union of conditions for both the main system and the constraint system to be strongly hyperbolic, and for all speeds to be physical $(|\lambda| \leq 1)$, is

$$
0<\sigma \leq 1, \quad 0<a b \leq 1, \quad 0<\chi \leq 1,
$$

and either $\chi \neq \sigma$, or $\chi=\sigma=\eta$. The union of all speeds is

$$
\lambda=\{0, \pm 1, \pm \sqrt{a b}, \pm \sqrt{\sigma}, \pm \sqrt{\chi}\} .
$$

We see that we can make all of these speeds either zero or one by choosing

$$
a b=\sigma=\chi=1 \Rightarrow c\left(1-a^{\prime} b\right)=0,
$$

that is, either $c=0$ or $a^{\prime}=a$. From now on we restrict ourselves to the choice $c=0$. This makes $\chi=1$, and removes $a^{\prime}$ from the system. We keep $a, b, \rho$ and $\sigma$ free, with the single restriction that $\sigma=1$ requires $a b=1$. The value of $\rho$ is irrelevant for strong hyperbolicity. Our results on strong hyperbolicity of the NOR system confirm and generalise those of [2], which were obtained using a pseudo-differential reduction to first order.

\section{Main system energy}

The most general ansatz for the energy density $\epsilon$ is quadratic in $\gamma_{i j, k}, K_{i j}$ and $f_{i}$. As well as contracting the free indices on pairs of these, we can form the contractions $K, d_{i} \equiv \gamma_{i j}{ }^{j}$ and $t_{i} \equiv \gamma^{j k} \gamma_{j k, i}$ first:

$$
\begin{aligned}
\epsilon= & c_{0} K_{i j}{ }^{2}+c_{1} f_{i}{ }^{2}+c_{2} t_{i}{ }^{2}+c_{3} d_{i}{ }^{2} \\
& +c_{4} d_{i} f^{i}+c_{5} t_{i} f^{i}+c_{6} d_{i} t^{i}+c_{7} K^{2} \\
& +c_{8} \gamma_{i j, k}{ }^{2}+c_{9} \gamma_{i j, k} \gamma^{i k, j} .
\end{aligned}
$$

The most general form that is conserved depends on four free parameters $c_{0}, c_{1}, c_{3}$ and $c_{7}$ obeying

$$
2(a b-1) c_{7}=(\sigma-2 a b+1) c_{0} .
$$

for arbitrary $(\rho, \sigma, a, b)$. The coefficient $c_{0}$ must be strictly positive and therefore there are two possibilities: either $a b=1$ (which now implies $\sigma=1$ ) and then $c_{0}$ 
and $c_{7}$ are independent, or $a b \neq 1$ and then $c_{7}$ is determined by $c_{0}$ and the parameters $(\sigma, a, b)$. What follows is valid in both cases, with the corresponding restrictions. Note that strong hyperbolicity and energy conservation together give the two directions of $\sigma=1 \Leftrightarrow a b=1$.

With the shorthands

$$
\begin{aligned}
\epsilon_{0}= & K_{i j}{ }^{2}+\frac{1}{4} \gamma_{i j, k}^{2}+\frac{2 a-a b-1}{2} \gamma_{i j, k} \gamma^{i k, j} \\
& -a d_{i}\left[f^{i}-\left(b-\frac{\rho}{2}\right) t^{i}\right] \\
\epsilon_{1}= & {\left[f_{i}-\left(b-\frac{\rho}{2}\right) t_{i}+(b-1) d_{i}\right]^{2}, } \\
\epsilon_{2}= & d_{i}^{2}-\gamma_{i j, k} \gamma^{i k, j} \\
\epsilon_{3}= & K^{2}+\frac{2+2 a b-2 a \rho+\sigma}{4} t_{i}^{2} \\
& +(a-1) d_{i} t^{i}-a t_{i} f^{i}
\end{aligned}
$$

(the indices on $\gamma_{i j, k}$ are raised only after differentiation), the energy can be written as

$$
\epsilon=c_{0} \epsilon_{0}+c_{1} \epsilon_{1}+\left[c_{3}-(b-1)^{2} c_{1}\right] \epsilon_{2}+c_{7} \epsilon_{3} .
$$

The flux is

$$
F^{i}=c_{0} F_{0}^{i}+\left[c_{3}-(b-1)^{2} c_{1}\right] F_{2}^{i}+c_{7} F_{3}^{i},
$$

with

$$
\begin{aligned}
F_{0}^{i}= & 2 a K^{i j}\left[f_{j}-(b-1) d_{j}-(b-\rho / 2) t_{j}\right] \\
& +\gamma^{i l} K^{j k}\left[2(1+a b-2 a) \gamma_{l j, k}-\gamma_{j k, l}\right], \\
F_{2}^{i}= & 4 \gamma^{i l} K^{j k}\left(\gamma_{l j, k}-\gamma_{l j} d_{k}\right), \\
F_{3}^{i}= & K\left[2 a f^{i}+(a \rho-2-\sigma) t^{i}+2(1-a) d^{i}\right] \\
& +2(1-a b) K^{i j} t_{j} .
\end{aligned}
$$

(As in BSSN, $\epsilon_{1}$ has no flux.) The flux in terms of characteristic variables is

$$
\begin{aligned}
F^{n}= & \frac{c_{0}}{2}\left(U_{+A B}^{2}-U_{-A B}^{2}\right)+\frac{c_{0}}{4 \sqrt{a b}}\left(U_{+A}^{2}-U_{-A}^{2}\right) \\
& +\frac{c_{0}+c_{7}}{2 \sqrt{\sigma}}\left(V_{+}^{2}-V_{-}^{2}\right)+\frac{c_{0}}{16} \frac{c_{0}+3 c_{7}}{c_{0}+c_{7}}\left(U_{+}^{2}-U_{-}^{2}\right)
\end{aligned}
$$

The characteristic variables including transversal derivatives are

$$
\begin{aligned}
U_{ \pm}= & -\partial_{n} \gamma_{q q}-2 z_{2} \partial^{A} \gamma_{A n} \pm 2 K_{q q}, \\
V_{ \pm}= & a f_{n} \pm \sqrt{\sigma} K_{n n}+\frac{a \rho-\sigma-1}{2} \partial_{n} \gamma_{q q} \\
& +\frac{a \rho-\sigma-2 a}{2} \partial_{n} \gamma_{n n}+z_{3} \partial^{A} \gamma_{A n} \\
& +\frac{c_{7}}{2\left(c_{0}+c_{7}\right)}\left(-\partial_{n} \gamma_{q q}-2 z_{2} \partial^{A} \gamma_{A n} \pm 2 \sqrt{\sigma} K_{q q}\right), \\
U_{ \pm A}= & a f_{A}-a \partial_{n} \gamma_{A n} \pm 2 \sqrt{a b} K_{A n} \\
& +z_{3} \partial^{B} \gamma_{A B}+\partial_{A}\left(z_{4} \gamma_{n n}+z_{5} \gamma_{q q}\right), \\
U_{ \pm A B}= & \mp K_{A B}+\frac{1}{2} \partial_{n} \gamma_{A B} \\
& +z_{2}\left(\partial_{(A} \gamma_{B) n}-\frac{1}{2} q_{A B} \partial^{C} \gamma_{C n}\right),
\end{aligned}
$$

with speeds $\lambda=\{ \pm 1, \pm \sqrt{\sigma}, \pm \sqrt{a b}, \pm 1\}$, where we have defined the shorthands

$$
\begin{aligned}
& z_{1}=\frac{c_{3}-(b-1)^{2} c_{1}}{c_{0}}, \\
& z_{2}=2 a-a b-1-2 z_{1}, \\
& z_{3}=a-a b-2 z_{1} \\
& z_{4}=(2 a b-\sigma-1) / 2+1-2 a+a \rho / 2+2 z_{1}, \\
& z_{5}=(2 a b-\sigma-1) / 2+a(1-3 b+\rho) / 2-z_{1} .
\end{aligned}
$$

Note that the last term of (160) requires $c_{0}+c_{7} \neq 0$. We shall see below that this is always true.

\section{Constraint system energy}

The constraint energy is quadratic in $H_{0}, M_{i}$ and $G_{i, j}$. The most general form that is conserved has three free parameters $w_{0}, w_{1}$ and $w_{2}$ for arbitrary $(\rho, \sigma, a, b)$. It is

$$
\begin{aligned}
\epsilon_{c}= & w_{0} H_{0}^{2}+4 w_{1} M_{i}^{2}+\frac{a w_{1}}{b}\left(G_{i, j}\right)^{2} \\
& +w_{2} G_{i, j} G^{j, i}+2 \frac{w_{0}-w_{1}}{b} G^{i}{ }_{, i} H_{0} \\
& +\frac{w_{0}-(1+a b) w_{1}-b^{2} w_{2}}{b^{2}}\left(G^{i}{ }_{, i}\right)^{2}
\end{aligned}
$$

The flux is

$$
\begin{aligned}
F_{c}^{i}= & -4\left(a w_{1}+b w_{2}\right) G^{j}{ }_{, j} M^{i}-4 w_{1} H_{0} M^{i} \\
& +4\left(b w_{2} G^{i, j}+a w_{1} G^{j, i}\right) M_{j} .
\end{aligned}
$$

In terms of characteristic variables,

$$
F_{c}^{n}=\frac{w_{1}}{2}\left(C_{+}^{2}-C_{-}^{2}\right)+\frac{w_{1}}{2 \sqrt{a b}}\left(C_{+A}^{2}-C_{-A}^{2}\right)
$$

where, including transversal derivatives,

$$
\begin{aligned}
C_{ \pm} & =H_{0}+\left(a+\frac{b w_{2}}{w_{1}}\right) \partial_{A} G^{A} \mp 2 M_{n}, \\
C_{ \pm A} & =a \partial_{n} G_{A}+\frac{b w_{2}}{w_{1}} \partial_{A} G_{n} \pm 2 \sqrt{a b} M_{A},
\end{aligned}
$$

with speeds $\lambda=\{ \pm 1, \pm \sqrt{a b}\}$.

\section{E. Symmetric hyperbolicity and causal speeds}

Positivity of the constraint energy requires that for a given value of $a b$ we must choose $w_{0}, w_{1}$ and $w_{2}$ obeying

$$
\begin{gathered}
w_{0}>0, \quad 0<\frac{w_{1}}{w_{0}}<1, \quad b^{2} \frac{w_{2}}{w_{1}}<\frac{3}{4}\left(1-\frac{w_{1}}{w_{0}}\right), \\
b^{2} \frac{\left|w_{2}\right|}{w_{1}}<a b<\frac{3}{2}\left(1-\frac{w_{1}}{w_{0}}\right)-b^{2} \frac{w_{2}}{w_{1}} .
\end{gathered}
$$

The positivity conditions of the main energy will be analysed separately for the generic and special cases. 
In the general case $a b \neq 1$ and $\sigma \neq 1$ positivity of the main energy requires that (using the strong hyperbolicity condition $\sigma>0$ and the causal speeds condition $\sigma \leq 1$ )

$$
\begin{aligned}
& c_{0}>0, \quad \frac{1+3 \sigma}{4}<a b<1, \\
& a^{2} c_{0}(1-\sigma)<2 \sigma c_{1}(1-a b), \\
& -\frac{5 c_{0}}{4} \frac{2 \sigma c_{1}(1-a b)-a^{2} c_{0}(1-\sigma)}{2 c_{1}(1-a b)(4-4 a b+3 \sigma)-a^{2} c_{0}(4 a b-1-3 \sigma)} \\
& <c_{3}-(b-1)^{2} c_{1}+\frac{1+2 a b-4 a}{4} c_{0}<0 .
\end{aligned}
$$

The value of $\rho$ is irrelevant also for symmetric hyperbolicity. The restrictions on $\sigma$ and $a b$ guarantee that $c_{0}+c_{7}$ and $c_{0}+3 c_{7}$ are always strictly positive. A simple example is

$$
\begin{aligned}
& a=\frac{3}{4}, \quad b=1, \quad \sigma=\frac{1}{2}, \quad \rho=\frac{2}{3}, \\
& c_{0}=1, \quad c_{1}=2, \quad c_{3}=c_{7}=0, \\
& w_{0}=1, \quad w_{1}=\frac{1}{4}, \quad w_{2}=0 .
\end{aligned}
$$

In the special case $a b=\sigma=1$ positivity of the main energy requires

$$
\begin{aligned}
& c_{0}>0, \quad c_{0}+3 c_{7}>0, \quad b^{2} c_{1}>c_{0}+c_{7}>0, \\
& -\frac{5 c_{0}}{4} \frac{b^{2} c_{1}-c_{0}-c_{7}}{3 b^{2} c_{1}-c_{0}-3 c_{7}} \\
& <c_{3}-(b-1)^{2} c_{1}+\frac{3 b-4}{4 b} c_{0}<0 .
\end{aligned}
$$

A simple example is

$$
\begin{aligned}
& a=b=\sigma=1, \quad \rho=\frac{2}{3}, \\
& c_{0}=1, \quad c_{1}=3, \quad c_{3}=c_{7}=0, \\
& w_{0}=1, \quad w_{1}=\frac{1}{4}, \quad w_{2}=0 .
\end{aligned}
$$

\section{CONSTRAINT-PRESERVING BOUNDARY CONDITIONS}

\section{A. The boundary system}

We have proved symmetric hyperbolicity for both the main system and the constraint system of the BSSN and NOR formulations. Therefore both formulations admit constraint-preserving boundary conditions of the type proposed in [10]. A general discussion is left to future work. Here we summarize the basic idea, and propose a family of boundary conditions for the case of a smooth boundary with tangential shift.

Because the constraint system is compatible with the evolution system, for every pair of characteristic variables $C_{ \pm}$of the constraint system with speeds $\pm \lambda$, there is a pair of characteristic variables $U_{ \pm}$with the same speeds, such that (after suitable normalisation), they obey

$$
C_{ \pm}=\partial_{n} U_{ \pm}+\ldots
$$

where the dots, here and in the remainder of this subsection, indicate transversal derivatives and lower-order terms. In order to guarantee that the constraint energy does not grow, we formally impose the homogeneous maximally dissipative boundary condition

$$
C_{+}-\kappa C_{-}=0
$$

on the constraint system. This must then be translated into a boundary condition on the main system. From (29), we have

$$
\partial_{t} U_{ \pm}=\left( \pm \lambda \alpha+\beta_{n}\right) \partial_{n} U_{ \pm}+\ldots
$$

In the following we restrict consideration to the case where $\beta_{n}=0$ on the boundary, so that the $\lambda=0$ characteristic variables propagate along the boundary. (183) is then equivalent to

$$
\partial_{t} U_{+}+\kappa \partial_{t} U_{-}=\ldots
$$

We define a variable $X \equiv U_{+}+\kappa U_{-}$that is restricted to the boundary, with evolution equation

$$
\partial_{t} X=\ldots
$$

and impose the boundary condition

$$
U_{+}+\kappa U_{-}=X
$$

on the main system.

We have chosen our notation in the previous sections so that in both BSSN and NOR we have

$$
\begin{aligned}
C_{ \pm} & =\partial_{n} U_{ \pm}+\ldots, \\
C_{ \pm A} & =\partial_{n} U_{ \pm A}+\ldots,
\end{aligned}
$$

and the boundary conditions for either system are

$$
\begin{aligned}
C_{+}-\kappa_{1} C_{-} & =0 \\
C_{+A}-\kappa_{2} C_{-A} & =0 \\
V_{+}-\kappa_{3} V_{-} & =F \\
U_{+A B}-\kappa_{4} U_{-A B} & =F_{A B},
\end{aligned}
$$

where $F$ and $F_{A B}$ are free boundary data, and (190 191) are implemented as

$$
\begin{aligned}
U_{+}+\kappa U_{-} & =X, \\
U_{+A}+\kappa U_{-A} & =X_{A} .
\end{aligned}
$$

In general the boundary system is coupled to the bulk system, so that $X$ and $X_{A}$ in (194 195) cannot be considered as given a priory, and then the constraint-preserving boundary conditions are not true maximally dissipative boundary conditions. There are two exceptions, which 
have been called "Neumann" and "Dirichlet" boundary conditions in the literature, where an extended boundary system can be given that decouples from the bulk system. This happens for the Einstein-Christoffel system linearised around Minkowski spacetime, [10], the full Einstein equations in harmonic gauge 11], and the Maxwell equations 13. This boundary system can then be evolved before the bulk system is evolved, $X$ and $X_{A}$ can be treated as given a priori, and the constraintpreserving boundary conditions become true maximally dissipative boundary conditions. The details, assuming a smooth boundary with tangential shift, are given in Appendices [B] and $[\mathrm{C}$ However, we would like to stress that these boundary conditions are very restrictive: it does not seem very physical to find the boundary of the spacetime without knowing what is inside.

As we have assumed that the shift is everywhere tangential to the boundary, and this is possible in the case of a non-smooth boundary (for example a cube) only for zero shift, we also restrict the analysis in this paper to a smooth boundary.

\section{B. Mode analysis}

If the boundary system does not decouple, we cannot use our current energy estimates to prove well-posedness of the initial-boundary value problem. We can, however, check a necessary condition for well-posedness, namely that there are no modes that grow exponentially in time where the growth rates increases unboundedly with spatial frequency. We conjecture that this condition is also sufficient.

In the frozen coefficients approximation that we have been using throughout this paper we assume that the linearised perturbation varies over space and time scales much smaller than those given by the background solution and the numerical domain. For consistency we must therefore assume that the domain is a half space and that the boundary is a plane. We introduce coordinates so that the domains is $-\infty<x^{1} \leq 0$ and $-\infty<x^{A} \equiv\left(x^{2}, x^{3}\right)<\infty$, and the metric in these coordinates is $\delta_{i j}$. In the frozen coefficient approximation $\alpha>0$ and $\beta^{i}$ are also constant in space and time. (As before we assume $\beta_{n}=0$ on the boundary, and therefore everywhere in the frozen coefficient approximation).

After a Fourier transform in $x^{A}$ and Laplace transform in $t$ we are left with a system of linear ODEs with constant coefficients in $x^{1}$. In general this can be transformed into a matrix eigenproblem by an exponential ansatz in $x^{1}$. (A priori an exponential times a polynomial in $x^{1}$ could be required to find the general solution but this is not the case here.) The general solution with homogeneous boundary conditions can therefore be written as a sum of modes of the form

$$
u\left(x^{1}, t\right)=e^{\left(\alpha s+i \beta^{A} \omega_{A}\right) t+i \omega_{A} x^{A}+\mu x^{1}} \bar{u}
$$

where $\bar{u}$ is a constant vector. With this ansatz we have $\partial_{0} u=s u, \partial_{n} u=\mu u$ and $\partial_{A} u=i \omega_{A} u$.

If the initial data are nonzero, we must add to the sum over modes a particular integral that does not concern us here because it is controlled by the boundary data 4]. We are interested only in modes that are squareintegrable over space at any moment in time. Therefore we assume that $\omega_{A}$ is real, and that $\operatorname{Re} \mu>0 . s$ and $\mu$ will in general be complex. If a mode of this form exists for some $\left(s, \omega_{A}, \mu, \bar{u}\right)$, then one exists also for $\left(k s, k \omega_{A}, k \mu, \bar{u}\right)$ for any $k>0$. Therefore, if any growing mode, with Re $s>0$, exists, there are growing modes with arbitrarily large growth rates and the problem is ill-posed. A necessary condition for well-posedness of the initial-boundary value problem is therefore that the homogeneous boundary conditions rule out the existence of any mode with $\operatorname{Re} s>0$ for real $\omega_{A}$ and $\operatorname{Re} \mu>0$.

For simplicity we concentrate again on NOR with (179. 181). $u=\left(\gamma_{i j}, K_{i j}, f_{i}\right)$ is decomposed with respect to the normal vector $n_{i}=(1,0,0)$. It is helpful to introduce the notation $f_{m} \equiv i \omega^{A} f_{A}$ and $f_{p} \equiv p^{i} f_{i}$ where $p^{i}$ is orthonormal to $\omega_{i}$ and $n_{i}$, and similarly for other tensor components. Substituting the ansatz (196) into the NOR evolution equations, we find after some linear algebra that, for $s \neq 0$,

$$
\left(s^{2}+\omega^{2}-\mu^{2}\right) \bar{\gamma}_{i j}=0
$$

and

$$
\begin{aligned}
\bar{K}_{i j} & =-\frac{s}{2} \bar{\gamma}_{i j}, \\
\bar{f}_{p} & =0 \\
\bar{f}_{m} & =-\frac{2}{3} \omega^{2}\left(\bar{\gamma}_{n n}+\bar{\gamma}_{q q}\right), \\
\bar{f}_{n} & =\frac{2}{3} \mu\left(\bar{\gamma}_{n n}+\bar{\gamma}_{q q}\right) .
\end{aligned}
$$

For a non-zero solution to exist, we must have

$$
\mu^{2}-s^{2}=\omega^{2} \text {. }
$$

The coefficients $\bar{\gamma}_{i j}$ are then free parameters. They determine the coefficients $\bar{K}_{i j}$ and $\bar{f}_{i}$ through (198 201).

Similarly, for the BSSN system with (122 123) we find the equivalent of condition (197) for $\overline{\tilde{\gamma}}_{i j}$ and $\bar{\phi}$, and

$$
\begin{aligned}
\bar{K} & =-6 s \bar{\phi} \\
\overline{\tilde{A}}_{i j} & =-\frac{s}{2} \overline{\tilde{\gamma}}_{i j}, \\
\overline{\tilde{\Gamma}}_{p} & =0 \\
\overline{\tilde{\Gamma}}_{m} & =-8 \omega^{2} \bar{\phi}, \\
\overline{\tilde{\Gamma}}_{n} & =8 \mu \bar{\phi} .
\end{aligned}
$$

Note that $s \overline{\tilde{\gamma}}_{i j}$ represents the time derivative of $\tilde{\gamma}_{i j}$. Therefore, as we assume that the algebraic constraint $D=0$ is being imposed continuously, $\overline{\tilde{\gamma}}_{i j}$ must be tracefree. Similarly, from the algebraic condition $T=0, \overline{\tilde{A}}_{i j}$ is also tracefree. With (197) obeyed, $\overline{\tilde{\gamma}}_{i \underline{j}}$ and $\bar{\phi}$ are free coefficients, and determine $\overline{\tilde{A}}_{i j}, \bar{K}$ and $\tilde{\Gamma}^{i}$. 
We now substitute the ansatz (1961) with these coefficients into the six constraint equations (190 193). We obtain six algebraic equations that are linear in the four components $\bar{\gamma}_{n n}, \bar{\gamma}_{n m}, \bar{\gamma}_{n p}, \bar{\gamma}_{q q}$ of $\bar{\gamma}_{i j}$ and the two components, $\bar{\gamma}_{m m} \equiv-\bar{\gamma}_{p p}, \bar{\gamma}_{m p}$ of the tracefree transversal object $\bar{\gamma}_{A B}$. We can solve these recursively to find that all $\bar{\gamma}_{i j}=0$, as long as

$$
\left(1-\kappa_{i}\right) \mu+\left(1+\kappa_{i}\right) s \neq 0 .
$$

for all four $\kappa_{i}$. For a mode to exist, this inequality must be violated for at least one of the $\kappa_{i}$. Let the value of this $\kappa_{i}$ be $\kappa$, which therefore obeys

$$
\kappa(\mu-s)=(\mu+s) .
$$

We now investigate the space of possible solutions $(\mu, s, \omega, \kappa)$ of the two algebraic equations (202) and (209) with $\operatorname{Re} \mu>0$ and $\operatorname{Re} s>0$, with the aim of finding a condition on $\kappa$ that excludes all such solutions. We first consider the case $\omega=0$. Then either $\mu=s=0$, or $\mu=-s$ and $\kappa=0$. Either solution does not correspond to growing square-integrable modes. We can now assume $\omega>0$, and parameterise all solutions by $s$. We find

$$
\mu(s)=\sqrt{s^{2}+\omega^{2}}, \quad \kappa(s)=\left(\frac{\mu-s}{\omega}\right)^{-2} .
$$

We choose the principal branch of the square root, because it maps $\operatorname{Re} s>0$ to $\operatorname{Re} \mu>0$, that is, the growing modes are precisely the square-integrable modes. This choice also maps $\operatorname{Re} s>0$ to $|\kappa|>1$. Therefore, we exclude all growing square-integrable modes if we restrict $\left|\kappa_{i}\right| \leq 1$ for all four $\kappa_{i}$, or $-1 \leq \kappa_{i} \leq 1$ for real $\kappa_{i}$. More details are given in Appendix D

\section{CONCLUSIONS}

We have constructed families of generalisations of the BSSN and NOR variants of the ADM evolution equations that are symmetric hyperbolic in the sense defined for second-order systems in [13]. This confirms the previous result of [20] on the BSSN equations, without recourse to any first-order reduction, and generalises it by finding the most general energies for the main and constraint system. This generalisation allows all characteristic modes to propagate with causal speeds, in particular with speeds $(0, \pm 1)$ only.

In our analysis of the BSSN equations we also clarify the role for hyperbolicity of imposing the algebraic constraints $\operatorname{det} \gamma_{i j}=1$ and $\operatorname{tr} \tilde{A}_{i j}=0$ during the evolution. We find that the equations can be made symmetric hyperbolic if these constraints are imposed continuously, and strongly hyperbolic without imposing the constraints, but adding an extra term to the evolution equations.

There is numerical evidence that densitising the lapse and imposing the trace constraint improves stability in moving single black hole simulations, even without imposing maximally dissipative constraint-preserving or boundary conditions 23]. This is not surprising, as these changes make the evolution equations strongly hyperbolic, and imposing the determinant constraint as well would make them symmetric hyperbolic.

Our results go some way towards explaining why the BSSN system has been relatively successful in simulating black hole or neutron star binaries. It is possible that the Bona-Massó formulation 24], a strongly hyperbolic first-order version of the Einstein equations that introduced variables similar to the $\tilde{\Gamma}^{i}$ or $f_{i}$, has not been as successful because it is first order, which we expect makes it more susceptible to constraint-violating instabilities of the convergent type.

The NOR system is basically the BSSN system without the conformal-traceless decomposition, and the similarity of our results for the two systems suggests that the NOR system shares all the advantages of the BSSN system, without the overhead of the extra variables $K$ and $\phi$ and extra constraints $T=0$ and $D=0$.

With symmetric hyperbolicity, we can make the initialboundary value problem formally well-posed by imposing maximally dissipative boundary conditions. However, these boundary conditions are in general not compatible with the constraints, and so large constraint violations (of the convergent type) propagate in from the boundaries. This can be avoided by replacing some of the maximally dissipative boundary conditions on the main system by maximally dissipative boundary conditions on the constraint system 10], and we have given details of how to do this for NOR and BSSN, for the case of a smooth boundary with tangential shift. Note that even when we have fixed the principal part of the field equations both the main and constraint energies still depend on a number of free parameters, which appear explicitly in the boundary conditions.

Except for two rather unphysical special cases, we have not proved that the initial-boundary value problem with constraint-preserving boundary conditions is well-posed. We have shown, however, that these boundary conditions rule out perturbation modes with unbounded growth, which is a key necessary condition for well-posedness [4]. We plan to investigate a proof of well-posedness, and in parallel to examine stability in numerical experiments.

Our discussion of both maximally dissipative and constraint-preserving boundary conditions assumes that the normal component $\beta_{n}$ of the shift vanishes at the boundary, as then the $\lambda=0$ characteristic modes propagate along the boundary. This restriction allows for a shift that is everywhere tangential to a smooth boundary, and this could be used for example to employ corotating coordinates in the simulation of a binary system. The case of a general shift will be investigated in future work.

All equations in this paper were derived using xTensor, an open-source Mathematica package for abstract tensor calculus, developed by JMM. It is available under the GNU Public Licence from 
http://metric.imaff.csic.es/Martin-Garcia/xAct/

\section{Acknowledgments}

The authors would like thank G Nagy, O Ortiz and O Reula for communicating a draft paper and for discussions. CG would like to thank the Kavli Institute for Theoretical Physics for hospitality while this work was begun, and T Baumgarte, G Calabrese, H Friedrich, L Kidder, L Lindblom, O Sarbach, M Scheel, D Shoemaker and J Vickers for helpful discussions. JMM was supported by the Comunidad Autónoma de Madrid and Fondo Social Europeo and also in part by the Spanish MCYT under the research project BFM2002-04031-C0202 .

\section{APPENDIX A: THE KST FORMULATION}

The Kidder-Scheel-Teukolsky (KST) formulation [7] is based on a reduction to first order of the ADM evolution equations with a densitised lapse (DADM) with the auxiliary variables $d_{k i j} \equiv \partial_{k} \gamma_{i j}$. This gives rise to the auxiliary constraints $C_{i j k l} \equiv \partial_{[i} d_{j] k l}=0$. The principal part of the evolution equations is

$$
\begin{aligned}
\partial_{0} \gamma_{i j} & \simeq-2 K_{i j} \\
\partial_{0} K_{i j} & \simeq \mathrm{DADM}+\gamma \gamma_{i j} H+\zeta \gamma^{k l} C_{k(i j) l} \\
\partial_{0} d_{k i j} & \simeq \mathrm{DADM}+\eta \gamma_{k(i} M_{j)}+\chi \gamma_{i j} M_{k}
\end{aligned}
$$

This system can be made strongly or symmetric hyperbolic for certain ranges of the parameters $\sigma, \gamma, \zeta, \eta$ and $\chi$. In particular, the Einstein-Christoffel (EC) system is the case $\gamma=0, \zeta=-1, \eta=4, \chi=0$, densitizing the lapse with $\sigma=1$ in our notation. In this Appendix we want to point out that only $\sigma$ and $\gamma$ have counterparts in a second-order system. $\zeta$ has a similar function to our parameter $a$, and $2 \eta+\chi$ has a similar function to our parameter $b$, but these parameters vanish if we replace $d_{k i j}$ by $\partial_{k} \gamma_{i j}: C_{i j k l}$ then vanishes identically, and $d_{i j k}$ is no longer evolved explicitly by (A3), but only implicitly by (A1). Comparing the KST system to NOR with the benefit of hindsight, one could say that the only indispensable effect of $d_{k i j}$ is to introduce the divergence $d_{k j}^{k}$ as an auxiliary variable.

\section{APPENDIX B: DIRICHLET BOUNDARY SYSTEM}

With

$$
-\kappa_{1}=\kappa_{2}=\kappa_{3}=\kappa_{4}=1
$$

we have (in our example NOR system)

$$
F=2 K_{n n}
$$

$$
\begin{aligned}
F_{A B} & =-2 K_{A B} \\
X & =4 K_{q q} \\
X_{A} & =2\left(f_{A}-\partial_{n} \gamma_{A n}\right)+(\rho-2) \partial_{A}\left(\gamma_{n n}+\gamma_{q q}\right) .
\end{aligned}
$$

The boundary system

$$
\begin{aligned}
\partial_{0} X & =\partial^{A} X_{A}-2 \partial^{A}\left(\partial_{A} \gamma_{q q}\right), \\
\partial_{0} X_{A} & =\frac{1}{2} \partial_{A} X+2 \partial_{A} F+2 \partial^{B} F_{A B}, \\
\partial_{0}\left(\partial_{A} \gamma_{q q}\right) & =-\frac{1}{2} \partial_{A} X
\end{aligned}
$$

decouples from the bulk system. Note that all variables of the boundary system have parity +1 under the reflection $n_{i} \rightarrow-n_{i}$ through the boundary. The boundary system is strongly hyperbolic with characteristic variables

$$
\begin{aligned}
X_{ \pm} & =4 \partial_{m} \gamma_{q q}-2 X_{m} \mp \sqrt{6} X \\
X_{0} & =X_{m}+\partial_{m} \gamma_{q q},
\end{aligned}
$$

which have speeds $\lambda=( \pm \sqrt{3 / 2}, 0)$, as well as $X_{p}$ and $\partial_{p} \gamma_{q q}$ with zero speed. (It is also symmetric hyperbolic, but this is not required for well-posedness if the boundary is smooth without boundary, and therefore we do not give details here.)

\section{APPENDIX C: NEUMANN BOUNDARY SYSTEM}

With

$$
-\kappa_{1}=\kappa_{2}=\kappa_{3}=\kappa_{4}=-1
$$

we have

$$
\begin{aligned}
F & =2 f_{n}-\partial_{n} \gamma_{n n}+(\rho-2) \partial_{n}\left(\gamma_{n n}+\gamma_{q q}\right), \\
U_{0}^{\prime} & =f_{n}+\frac{\rho-2}{2} \partial_{n}\left(\gamma_{n n}+\gamma_{q q}\right), \\
F_{A B} & =\partial_{n} \gamma_{A B} \\
X & =-2 \partial_{n} \gamma_{q q} \\
X_{A} & =4 K_{A n} .
\end{aligned}
$$

The autonomous boundary system is

$$
\begin{aligned}
\partial_{0} X= & \partial^{A} X_{A}, \\
\partial_{0} X_{A}= & \frac{1}{2} \partial_{A} X+2 \partial^{B} F_{A B}+2 \partial_{A} F \\
& -2 \partial_{A} U_{0}^{\prime}-2 \partial^{B} \partial_{B} \gamma_{A n}, \\
\partial_{0}\left(\partial_{A} \gamma_{B n}\right)= & -\frac{1}{2} \partial_{A} X_{B}, \\
\partial_{0} U_{0}^{\prime}= & 0 .
\end{aligned}
$$

Note that all variables of the boundary system have parity -1 . The boundary system is strongly hyperbolic with characteristic variables

$$
\begin{aligned}
X_{ \pm} & =4 \partial_{m} \gamma_{m n}-X \mp \sqrt{6} X_{m} \\
Y_{ \pm} & =\partial_{m} \gamma_{p n} \mp \frac{1}{2} X_{p} \\
X_{0} & =\partial_{m} \gamma_{m n}+\frac{1}{2} X
\end{aligned}
$$


with speeds $\lambda=( \pm \sqrt{3 / 2}, \pm 1,0)$, as well as the zero speed variables $U_{0}^{\prime}, \partial_{p} \gamma_{m n}$ and $\partial_{p} \gamma_{p n}$.

\section{APPENDIX D: DETAILS OF THE MODE ANALYSIS}

An alternative approach to finding the range of real values of $\kappa$ that exclude growing modes would be to solve (202) and (209) explicitly for the values of $s$ and $\mu$ that are allowed for a given $\kappa$, and then check if they correspond to a growing, square-integrable mode. For $\kappa=0$ we have $\omega=0$ and $\mu=-s$. Therefore $\operatorname{Re} \mu>0$ implies $\operatorname{Re} s<0$, and vice versa, and so there are no growing square-integrable modes. For each $\kappa \neq 0$ we find two values of $s$ and $\mu$. For $\kappa>0$, we can parameterise them as

$$
\begin{aligned}
D_{ \pm}: \quad s & = \pm \omega \sinh \varphi, & & -\infty<\varphi<\infty, \\
\Rightarrow \mu & = \pm \omega \cosh \varphi, & & \kappa=e^{-2 \varphi} .
\end{aligned}
$$

The solution $D_{+}^{+}$with the upper sign and $\varphi>0$ has $\operatorname{Re} s>$ and $\operatorname{Re} \mu>0$, and corresponds to growing squareintegrable modes. To exclude these, we must demand $\kappa \leq 1$. However, for $\kappa<0$ there is a potential fallacy in this purely algebraic approach.

Consider the complex $s$ plane. For definiteness we place branch cuts from $i \omega$ to $i \infty$ and from $-i \omega$ to $-i \infty$. Consider the two contours $C_{+}$and $C_{-}$, parameterised by $\varphi$, which wrap around the upper and lower branch cut respectively:

$$
\begin{aligned}
C_{ \pm}: \quad s & = \pm i \omega \cosh \varphi, & & -\infty<\varphi<\infty \\
\Rightarrow \mu & = \pm i \omega \sinh \varphi, & & \kappa=-e^{2 \varphi}
\end{aligned}
$$

These give us the two possible values of $\mu$ and $s$ for each real $\kappa<0$. From a point of view where we only consider real values of $\kappa$, none of these modes are growing or square-integrable, and so we do not seem to obtain any restriction on negative $\kappa$. However, we should consider modes with purely imaginary $\mu$ and $s$ as limiting cases of growing square-integrable modes as $\operatorname{Re} s \rightarrow 0_{+}$and $\operatorname{Re} \mu \rightarrow 0_{+}$. Clearly this is the case for the modes lying on the contours $C_{ \pm}^{+}$(i.e. $\varphi>0$ ) which are to the right of the branch cuts and therefore contiguous with $\operatorname{Re} s>0$, but not for the contours $C_{ \pm}^{-}$, which are to the left of the branch cut. For the modes on $C_{ \pm}^{+},|\kappa|>1$, and so they are suppressed by any boundary condition with $|\kappa| \leq 1$. This is the range of $\kappa$ that we expect from the energy method. Another way of seeing that the modes on $C_{ \pm}^{-}$ should not be considered is to note that our mode analysis is really derived from a Laplace transform in $t$ [4], so that we need a contour for the inverse Laplace transform that is to the right of all branch cuts (and singularities) in the $s$ plane.

The boundary of $\operatorname{Re} s>0$ is given by the union of the three contours $C_{-}^{+}, C_{0}$ and $C_{+}^{+}$where

$$
\begin{aligned}
C_{0}: \quad s & =i \omega \sin \varphi, \quad-\frac{\pi}{2}<\varphi<\frac{\pi}{2}, \\
\Rightarrow \mu & =\omega \cos \varphi, \quad \kappa=e^{2 i \varphi} .
\end{aligned}
$$

This contour can also be used as a contour for the inverse Laplace transform. All contours in the $s$-plane discussed here and their images in the $\kappa$ plane are shown in Fig. 1] The shaded areas are $\operatorname{Re} s>0$ and its image $|\kappa|>1$. Re $s$ is bounded by by the union of the three contours $C_{-}^{+}, C_{0}$ and $C_{+}^{+}$and $\operatorname{Re} \mu$ by their images. This proves the claim made above that $\operatorname{Re} s>0$ is mapped to $|\kappa|>1$.
[1] RM Wald, General Relativity, University of Chicago Press, 1984; and references therein.

[2] G Nagy, OE Ortiz and OA Reula, Strongly hyperbolic second order Einstein's evolution equation, e-print gr-qc/0402123

[3] G Calabrese et al, Phys. Rev. D 66, 041501 (2002).

[4] B Gustafsson, H-O Kreiss and J Oliger, Time-dependent problems and difference methods, John Wiley, New York 1995.

[5] S Frittelli and OA Reula, Phys. Rev. Lett. 76, 4667 (1996).

[6] A Anderson and YW York, Jr, Phys. Rev. Lett. 82, 4384 (1999).

[7] LE Kidder, MA Scheel and SA Teukolsky, Phys. Rev. D 64, 064017 (2001).

[8] OA Reula, Living Reviews in Relativity 1998-3.

[9] H Friedrich and G Nagy, Comm. Math. Phys. 201, 619 (1999).

[10] G Calabrese et al, Commun. Math. Phys. 240, 377 (2003).

[11] B Szilagyi and J Winicour, Phys. Rev. D 68, 041501 (2003).

[12] MA Scheel et al, Phys. Rev. D 66124005 (2002).
[13] C Gundlach and JM Martín-García, Symmetric hyperbolic form of systems of second-order evolution equations subject to constraints, e-print gr-qc/0402079

[14] M Shibata and T Nakamura, Phys. Rev. D 52, 5428 (1995).

[15] T Baumgarte and SL Shapiro, Phys. Rev. D 59, 024007 (1999).

[16] M Alcubierre et al, Phys. Rev. D 62, 124011 (2000).

[17] M Shibata and K Uryu, Prog. Theor. Phys. 107, 265 (2002).

[18] M Alcubierre et al, Phys. Rev. D 64061501 (2001).

[19] U Sperhake et al, Phys. Rev. D 69, 024012 (2004).

[20] O Sarbach, G Calabrese, J Pullin and M Tiglio, Phys. Rev. D 66, 064002 (2002).

[21] JW York, Jr, in Sources of Gravitational Radiation, ed. L Smarr, Cambridge UP 1979. v

[22] H-J Yo, TW Baumgarte and SL Shapiro, Phys. Rev. D 66, 084026 (2002).

[23] P Laguna and D Shoemaker, Class. Quantum Grav. 19, 3679 (2002)q .

[24] C Bona et al., Phys. Rev. Lett. 75, 600 (1995). 

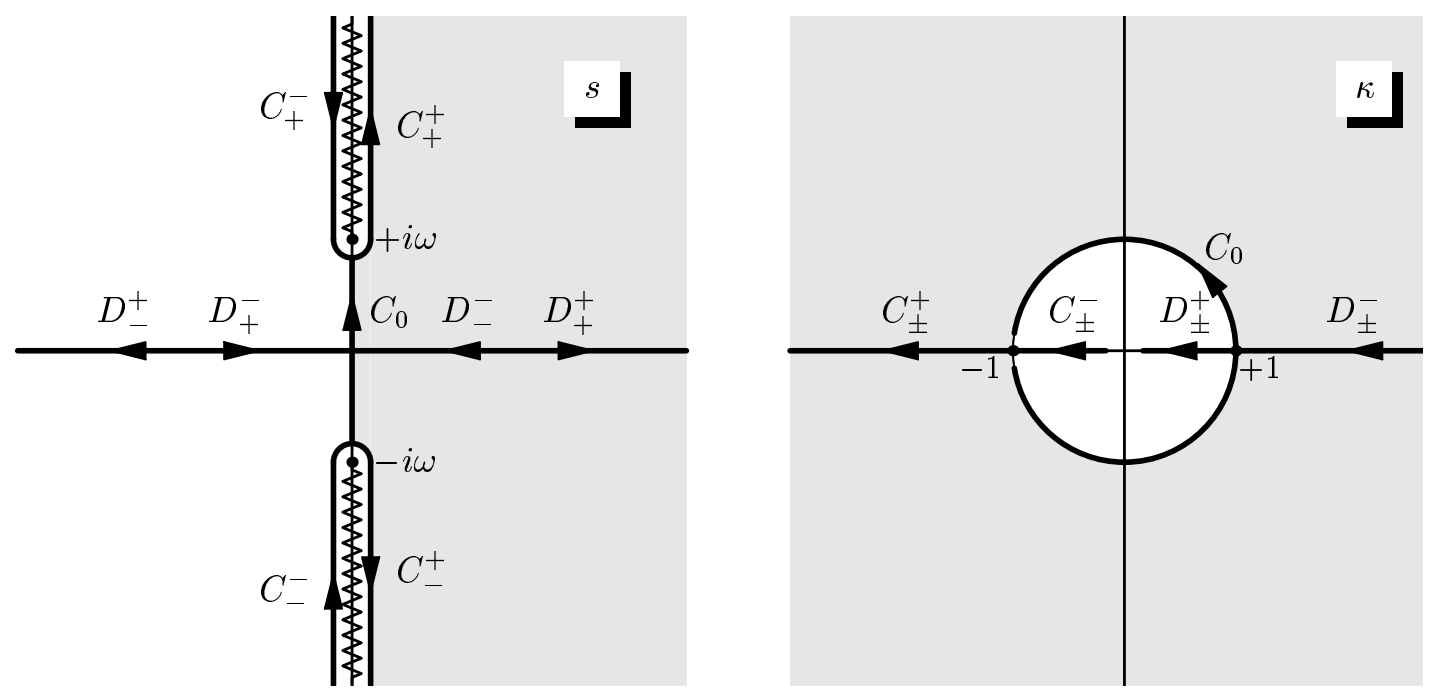

FIG. 1: Contours $C$ and $D$ in the complex $s$ plane and their images in the $\kappa$ plane under (210). Arrows point towards increasing parameter $\varphi$. The shaded area in the $s$ plane is mapped to the shaded area in the $\kappa$ plane. 